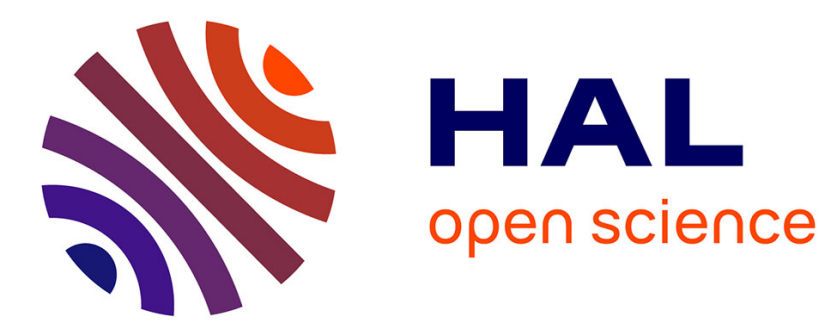

\title{
Efficient Broadcast of Alert Messages in VANETs
}

\author{
Hadjer Goumidi, Zibouda Aliouat, Makhlouf Aliouat
}

\section{To cite this version:}

Hadjer Goumidi, Zibouda Aliouat, Makhlouf Aliouat. Efficient Broadcast of Alert Messages in VANETs. 6th IFIP International Conference on Computational Intelligence and Its Applications (CIIA), May 2018, Oran, Algeria. pp.448-459, 10.1007/978-3-319-89743-1_39 . hal-01913913

\section{HAL Id: hal-01913913 \\ https://hal.inria.fr/hal-01913913}

Submitted on 7 Nov 2018

HAL is a multi-disciplinary open access archive for the deposit and dissemination of scientific research documents, whether they are published or not. The documents may come from teaching and research institutions in France or abroad, or from public or private research centers.
L'archive ouverte pluridisciplinaire HAL, est destinée au dépôt et à la diffusion de documents scientifiques de niveau recherche, publiés ou non, émanant des établissements d'enseignement et de recherche français ou étrangers, des laboratoires publics ou privés.

\section{(c)(1)}

Distributed under a Creative Commons Attribution| 4.0 International License 


\title{
Efficient Broadcast of Alert Messages in VANETs
}

\author{
Hadjer Goumidi ${ }^{1,2}$, Zibouda Aliouat ${ }^{1,2}$, Makhlouf Aliouat ${ }^{1,2}$ \\ ${ }^{1}$ University Ferhat Abbas Setif 1 \\ ${ }^{2}$ Laboratory of Networks and Distributed Systems \\ Algeria, Setif 19000 \\ hadjer.goumidi@univ-setif.dz, zaliouat@univ-setif.dz, \\ maliouat@univ-setif.dz
}

\begin{abstract}
VANET networks consist of several vehicles communicate with each other or with road fixed stations in order to offer a secure collaborative driving environment with the help of broadcasting emergency messages. These messages play a significant role in road safety by warning vehicles of any potential danger. The work reported in this paper is to devise an efficient broadcast protocol fulfilling alert message requirements in the VANETs context. Reacting in time to these urgent events may be important for crucial situations like road accidents.

Performance evaluation of our proposal EBAM is carried out through simulations with combination of VanetMobiSim and Network Simulator 2 and obtained results, in terms of loss rate, delay and reception rate of urgent messages, outperform those given by the two known referred protocols.
\end{abstract}

Keywords: Efficient Broadcast, Emergency message, Dependable delivery, VANETs.

\section{Introduction}

The technological development that has seen the world of today affects all areas, particularly the communication area which is experiencing a considerable evolution by the advances of wireless technology which has partially solved the problem of road accidents. Our work is coming in this context. In order to solve the problem of vehicle crashes, researchers think to exploit this technology enabling vehicles to establish direct (or indirect) links between them for being aware of potential critical situations like imminent accident or dangerous obstacles. This enabling technology is represented by VANET (Vehicular Ad hoc NETwork). Indeed, one of the promising applications of these networks is to allow vehicles equipped with specific sensors to detect the immediate environment, and alert drivers of vehicles around early in case of accident risks.

VANET as comfort communication can be made by two means: Periodic Safety Message (Referred in the sequel as Beacon) and Event Driven Message (referred as Emergency Message). Both message types share only one control channel. The Beacon messages are messages about status of sender vehicle. Status information includes position, speed, heading towards, etc $\cdots$ about the sender. 
Beacons provide resent or latest information of the sender vehicle to all present vehicles in the network, which help to know the position in the current network and anticipate the movement of vehicles.

Beacon messages are sent antagonistically to neighboring vehicles every 10 seconds. Emergency Messages are messages sent by a vehicle that detects a potential dangerous situation on the road; this information should be dispersed as alarm or alert to other vehicles pointing out a prospective danger that could affect the incoming vehicles. VANET is a high mobile or volatile network where nodes are kept changing their positions, moving in speed, which means that these vehicles may be get influence, even if they are very far from the danger. They can reach a danger in short time, so a fraction of second should be very important to avoid a danger [5]. Because of the importance of these messages exchanged between vehicles, it is necessary to ensure that these messages attain the largest number of vehicles in a minimum delay. These emergency messages are delivered in broadcasting way, where all the vehicles within the coverage area of the sender should receive the messages. The communication range of sender is not enough, due to the fading effects and attenuation; it hardly reaches a $1000 \mathrm{~m}$ (which is the DSRC communication range). Critical information should be received by vehicles, in time, far from the danger for avoiding risks. The prospect of message reception can reach $99 \%$ in short distances and can be decreased up to $20 \%$ at half of the communication range [7]. Therefore, it is necessary to provide a technique for raising the emergency message reception with high reliability and availability. The dissemination of the emergency messages poses several problems such as collisions, the problem of broadcast storm, problem of the hidden node etc. Several works such as PCBB, CBB, and EMDV... have been proposed to solve the problem of broadcasting emergency messages in VANETs.

\section{Related Works}

In [2], authors proposed a Dynamic Search-Assisted Broadcast (DSAB) protocol to efficient broadcast of emergency messages in VANETs. It based on controlling dynamically the transmission power to estimate the vehicle density and the use of n-way search mechanism to find the farthest vehicle in VANETs. The problem of this protocol is the choose of one vehicle which is the farthest one to rebroadcast the message because in VANET, vehicles always change their position and the farthest vehicle may do not receive the message, it may become out of range transmission when sending the message or it cannot receive the message because of the channel problems. A Distribution-Adaptive Distance with Channel Quality (DADCQ) protocol for efficient multi-hop broadcast of VANET is presented in [6]. It combines local spatial distribution information and other factors with the distance method heuristic to select rebroadcasting nodes. Authors in [4] presentd new distance-aware safety-related message broadcasting algorithm to enhance the propagation distance in the latency time of safety related message broadcasting. It generates the lenghts of backoff times by 
calculating the distances between the source node and its forwarding node. The farthest forwarding vehicle has the highest probability to forward messages. In [9], authors proposed an algorithm for data dissemination mechanism for motorway environment in VANETs. This protocol is based on an improved K-nearest neighbors prediction to predict the status of the nodes, and on construction of a forwarder set to avoid the broadcast storm problem. Limited Area-Based (LAB) scheme [3] adjusts the size of an area that contains the broadcasting nodes for obtaining a faster propagation time. Authors in [8] presented a counting-based broadcast model of emergency message dissemination in VANETs which is based on counting all possible cases of contention window assignments to all the vehicles simultaneously receiving a broadcast message. In [5], authors proposed Pso Contention Based Broadcast (PCBB) protocol that allows vehicles to increase the reception percentage of emergency information. In this protocol the network must be divided into several segments to help the sender to determine the next forwarder of the emergency message which is the furthest vehicle in the last non-empty segment.

The MIN is the lower bound of the last non-empty segment it calculated using the fitness function [5].

This protocol has a serious problem because the MIN calculation by fitness function is not always correct. In some cases, the MIN is greater than the max, this algorithm can be applied only in dense areas where the number of vehicles is large and the distance between them is small.

In [1], the authors proposed that the reception probability of the broadcast message instantly decreases at a distance greater than $66 \%$ of the transmission range, then they choose the forwarder as the node that has a distance equal to $66 \%$ of the transmission range.

To resolve the problem of the Protocol PCBB, we tried to apply this algorithm which chooses the node that has a distance equal to $66 \%$ of the transmission range of the transmitter as forwarder

When we choose the node $66 \%$, not the max (the farthest), the message takes a great reception time than that of the max. This algorithm does not use segmentation, so the forwarder has not potential nodes, and if the message fails to arrive to the forwarder it will be lost.

\section{THE PROPOSED PROTOCOL}

As a first step in our approach, we will present the EBAM (Efficient Broadcast of Alert Messages) protocol that ensures safe transmission of alert messages. From the disadvantages noted during the study of the protocols proposed in the specialized literature, we were able to identify an approach to avoid some problems detected in these protocols. The approach also assured high reliability avoiding collisions, decreasing the channel load with minimal cost and low response time. EBAM protocol can be used in all areas (dense and sparse networks) contrary to PCBB algorithm which can be applied only in dense areas.

The main idea of our protocol is to transmit the message to the farthest node 


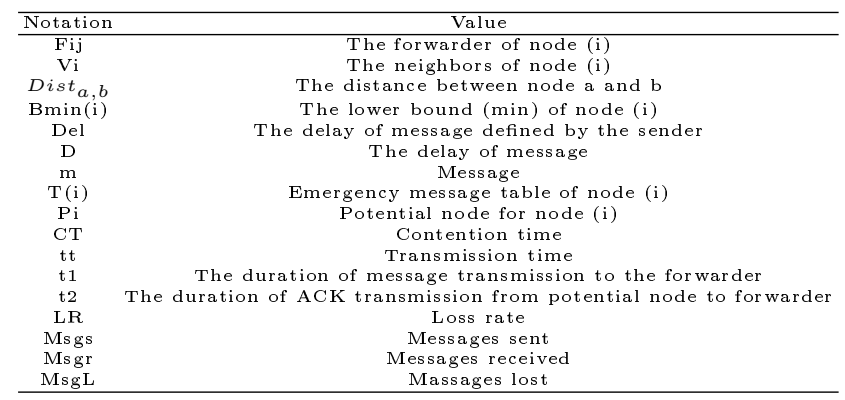

Table 1: Value notations table

within the transmission range. This node is defined as a forwarder. If the forwarder does not receive the message we resort to using potential nodes, such as the min of these nodes represents $60 \%$ of the farthest node distance.

We choose $60 \%$ as a lower bound because in [1] authors suppose that the distance $66 \%$ is the most appropriate to increase the reception rate.

Our approach has four steps:

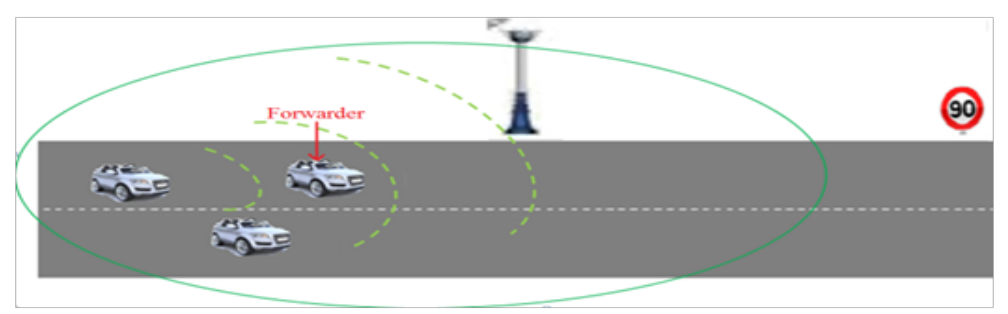

Fig. 1: Case of RSU further than forwarder.

\subsection{Preparing to send}

Each node $n_{i}$ knows the position of its neighbor nodes because of periodic security messages that are transmitted every second (beacon), allowing to calculate the distance between nodes to select the forwarder.

The selection of forwarder inspired from the PCBB protocol, which is the furthest vehicle in the last non-empty segment by using the formula (1):

$$
F_{i, j}=j / \max \left(\text { Dist }_{i, k}\right) ; k \in V_{i}
$$

The RSU sends its location to all nodes in its transmission range periodically, and each vehicle can calculate the distance between it and the RSU, for deciding 
which will receive the emergency message (forwarder or the RSU) by using the formula (2):

$$
\operatorname{Dist}_{i, j}=\min \left(\text { Dist }_{i, R S U}, \text { Dist }_{i, F_{i, j}}\right)
$$

In sparse area, we can found the case when the RSU is within transmission range of node (i) and it is farther than the forwarder as explain in example of Fig. 1.So, each vehicle must check if the RSU is within its transmission range, it will receive the message, else it sends the message to forwarder.

We calculate the min by using formula (3):

$$
\operatorname{Bmin}_{i}=\operatorname{Dist}_{i, F_{i, j}} \times 60 \%
$$

When a vehicle detects an emergency (e.g. accident), it creates an alert message ADV-ALERT, the format of this message is represented in Table 2. The emergency message contains:

Source identifier to avoid the problem of broadcast storm, we use this identifier which prevents the sender to retransmit the same message.

Message identifier We use this identifier to differentiate messages transmitted by the same sender. If the transmitter detects various accidents, it must send a various emergency messages, and vehicles must differentiate if this message is a new emergency message or it is a broadcast storm problem.

Priority identifier this identifier is used to classify the messages according to their importance in order to make it easier for the receiving vehicle to know the importance of the messages.

Forwarder ID We select the forwarder as the last vehicle in the last non-empty segment to avoid collisions and to transmit the message very quickly.

Transmission time and Delay The emergency message requires a short time, and the transmission of the emergency message after this time occupies the channel and loads the network without interest. The sender defines its message transmission time and the delay (Del) of this message.

Position the absence of characterization of the emergency event causes network load problem and can cause collisions leading to message loss and delay in reception. To solve this problem, the vehicle that detects the event sends the position of that event using GPS. 
Min it is necessary to send the min in the message, for each vehicle that receives this message to know if it is a potential node or not.

Data the emergency message must contain important data such as:

- If there are victims or wounded persons.

- The nature of the urgent case (accident, avalanche...).

\begin{tabular}{|l|l|l|l|l|l|l|l|}
\hline SourceID & MessageID & PriorityID & ForwarderID & Transmission time & Delay & Position & Min \\
\hline
\end{tabular}

Table 2: Format of the emergency message.

If a vehicle detects an event, it checks whether the same location exists in the emergency message table, if so, it does not creates the message, otherwise it creates the message and it adds the location of this event.

The use of the event position can help the emergency vehicles (ambulances) to intervene more quickly.

\subsection{The sending step}

After the creation of the emergency message $A D V-A L E R T$ and the selection of the forwarder, the sender broadcasts the message in the network to alert other vehicles about any potential danger.

\subsection{Receiving step}

Each vehicle existing in the transmission range of the sender that receives the message $A D V$-ALERT, checks if it is a potential node by using formula (4):

$$
S=\operatorname{Dist}_{i, j}-\operatorname{Bmin}_{j} ; \begin{cases}j \in P_{i}, & S \geqslant 0 \\ j \in V_{i}, & S<0\end{cases}
$$

The vehicles running in the last segment (potential nodes) initiate a contention time for waiting the forwarder to transmit a reply by using the formula (5) as explained in the example of Fig. 2.

$$
C T=t t+t_{1}+t_{2}+\varepsilon
$$

Then they detect the channel if no reply has been transmitted from the forwarder or other vehicles. It means that the forwarder has not received the message, so the first vehicle from the potential vehicles set that transmits the reply is defined as a forwarder. Once the reply is transmitted, the other vehicles 


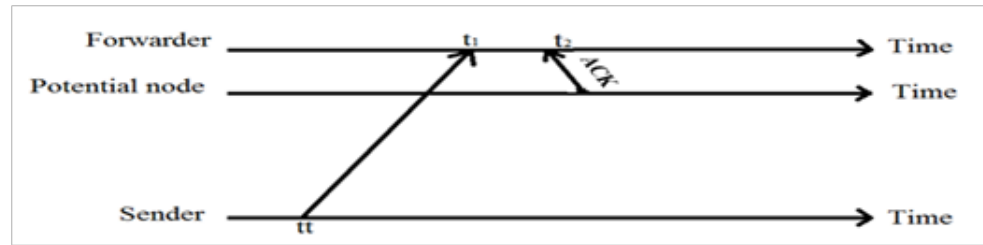

Fig. 2: Contention time illustration.

stop the transmission to avoid collisions. Fig. 3 depicts the receiving step.

Before sending the reply, the forwarder must check if it saved the $A D V$-ALERT in its emergency message table for sending the reply and for transmitting the $A D V$-ALERT to its forwarder. As shown in Fig. 4, it must check:

Priority ID: The forwarder checks the importance of the message if it is a big number, it does not save it, if none it checks the other indices (if they are acceptable) it saves the message in the table according to its priority level.

Transmission time and Delay: In general, the alert message is urgent and it does not take much time. Each vehicle receiving this message must calculate D using formula (6). To decide saving the message or not, in its emergency message table for the retransmission, the node uses the formula (7):

$$
\begin{gathered}
D_{m}=T r-T e \\
h=\text { Del }-D_{m}, \begin{cases}m \in T[i], & h \geqslant 0 \\
m \notin T[i], & h<0\end{cases}
\end{gathered}
$$

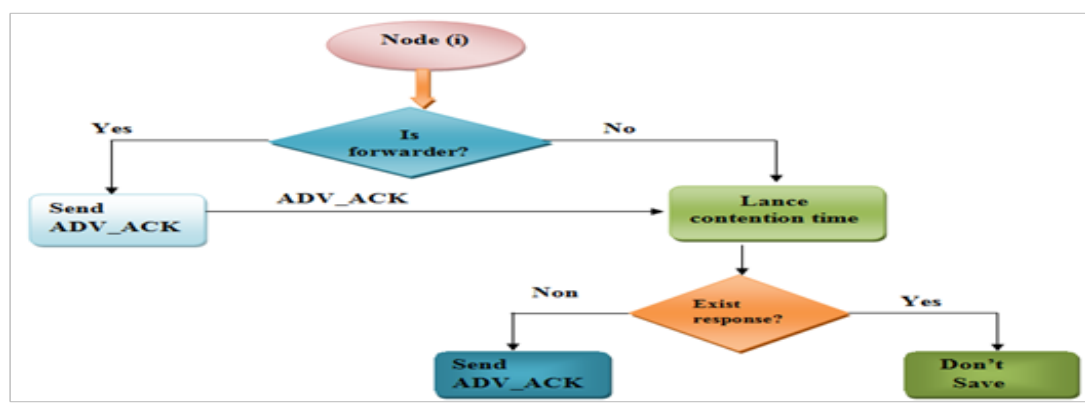

Fig. 3: Alert message reception flowchart.

After each 30s, each vehicle must update its table, the messages that exceed the delay will be deleted. 


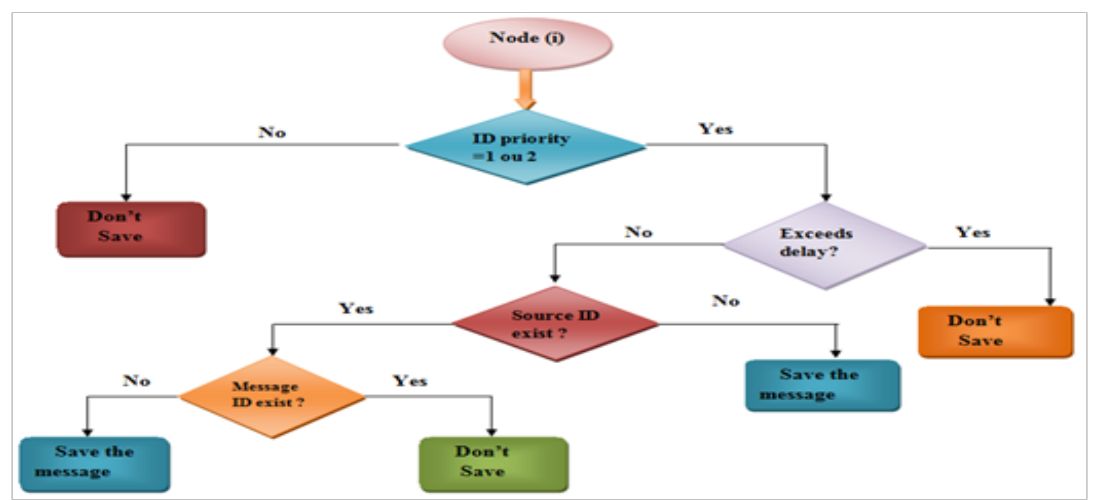

Fig. 4: Flowchart for checking message saving.

Source ID and Message ID are used to check if the message is transmitted before or not. If the forwarder found that the $A D V$-ALERT message has the same Source ID of a message stored in the emergency message table, it should check its message ID: if it found the same message ID then it does not save the message, otherwise the message will be saved.

\subsection{Rebroadcasting steps}

After saving the $A D V$-ALERT in emergency message table, The forwarder must broadcast it in its transmission range to its forwarder and follow the same steps defined previously.

\section{Simulation}

\subsection{Simulation parameters}

the simulation parametres are shown in table 3 .

Fig. 5 shows the number of messages transmitted, lost and received over the time for the $\mathrm{PCBB}$ protocol. We note that only one message is received the rest of messages are lost (the Min is greater than the max) until the end of the simulation.

Fig. 6 shows the number of messages transmitted, lost and received over the time for the $66 \%$ protocol. It is clear that the number of lost messages is great, only 8 messages among 307 sent messages are received and the rest is lost. This loss is due to the absence of potential nodes.

Fig. 7 shows the number of messages transmitted, lost and received over the time for EBAM proposal.

We note that in the proposed protocol the time that the message reach the RSU and the numbre of messages transmitted is less than in the PCBB and $66 \%$ 


\begin{tabular}{c|c}
\hline Topology $\left(\mathrm{m}^{2}\right)$ & $1000^{*} 1000$ \\
Number of nodes & 100 \\
Number of RSUs & 1 \\
Communication range of nodes & $300 \mathrm{~m}$ \\
Communication range of RSU & $1000 \mathrm{~m}$ \\
Speed $(\mathrm{m} / \mathrm{s})$ & 6 \\
MAC layer & MAC $802 \_11$ \\
Simulation Time(s) & $300 \mathrm{~s}$ \\
Mobility model & Random Following IDM_LC \\
\hline
\end{tabular}

Table 3: Simulation parameters.

protocols, this is due to the absence of lost messages practically. So the vehicle does not need to retransmit the message. At any time of simulation a packet sent can be not yet received but at the end of simulation all packets sent reached the destination.

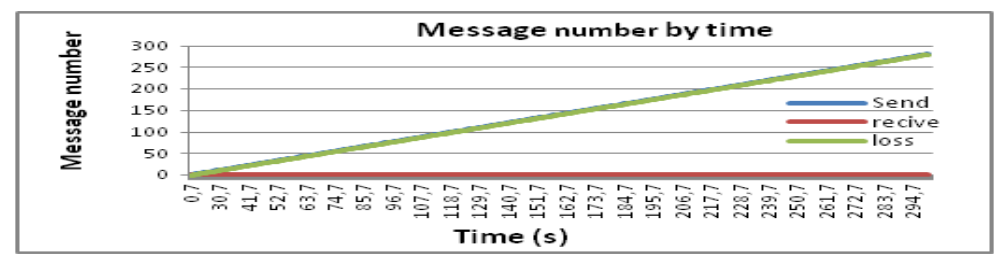

Fig. 5: Number of messages transmitted, received and lost in the PCBB protocol.

Fig. 8 shows messages loss rate according to the number of messages transmitted in the three protocols. We note that the messages loss rate in our protocol is approximately zero, and the two other protocols have a great loss rate compared to the EBAM, but the loss rate of the PCBB protocol is greater than in the $66 \%$ protocol.

Fig. 9 shows the reception delay of alert messages according to the three protocols. We calculate the reception delay of alert message by using the expression (7). From Fig. 9, we note that in EBAM, a message is received after 40.63 s from its transmission, but in the other two protocols the message does not arrive at the RSU (the simulation time is exhausted and the message did not achieve its destination). The loss rate of the three protocols is calculated by (8):

$$
M s g_{L}=\sum M s g_{s}-\sum M s g_{r} ; \quad L R=\frac{M s g_{L}}{\sum M s g_{s}}
$$




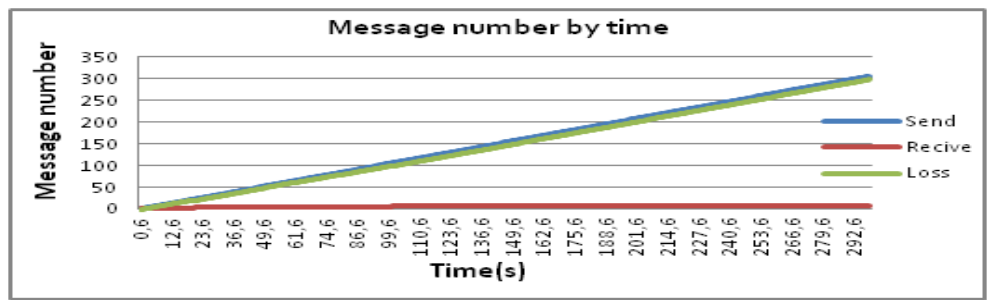

Fig. 6: Number of messages transmitted, received and lost in the $66 \%$ protocol

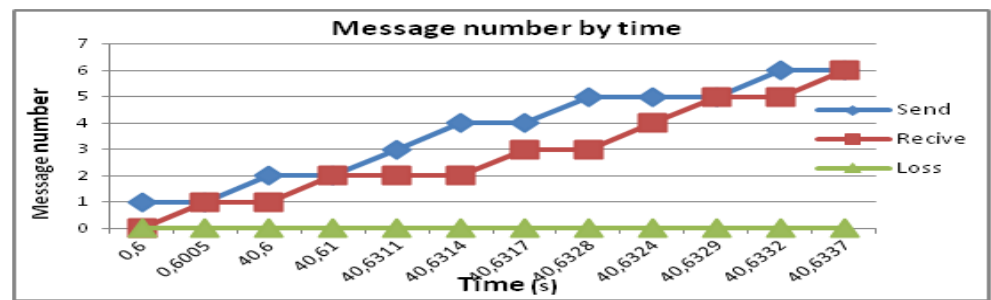

Fig. 7: Number of messages transmitted, received and lost in EBAM protocol.

\subsection{Evaluation of protocol performance}

For evaluating our protocol performance, we tested various scenarios by changing the parameters.

Fig. 10 represents the reception rate of the alert messages compared with the number of nodes according to the number of RSUs. In this figure we change the number of nodes and RSUs and the topology; we use, 50, 100, 200, 350 and 500 nodes, with 0,1 or 2 RSUs in topology of $2000^{*} 2000\left(\mathrm{~m}^{2}\right)$.

We remark that the reception rate is almost $20 \%$ when nodes broadcast the message between them. When one RSU broadcasts it, the reception rate is increased up to $80 \%$, and in the case where two RSUs broadcast the message, the reception rate reach approximately 100\%(the RSUs almost cover all the Topology).

Fig. 11 represents the simulation result of reception delay in second compared with the number of nodes. In this scenario we change only the number of nodes we use, 50, 100, 200, 350 and 500 nodes.

The reception delay is decreased with the increase of the number of nodes. This is due to the number of vehicles that transmit the message. When the number of vehicles becomes important, the message takes less reception time.

Fig. 12 represent the simulation result of reception delay in second related to the changing in speed. In this figure we change the speed parametre; we test speed of $6,12,20,40$ and $100 \mathrm{~m} / \mathrm{s}$ with fixed number of nodes (100 nodes).

The reception delay decreaseed up with the increase of speed due to the speeds of the vehicles transmitting the message. When the speed of the vehicles is important the message takes less time to be received. 


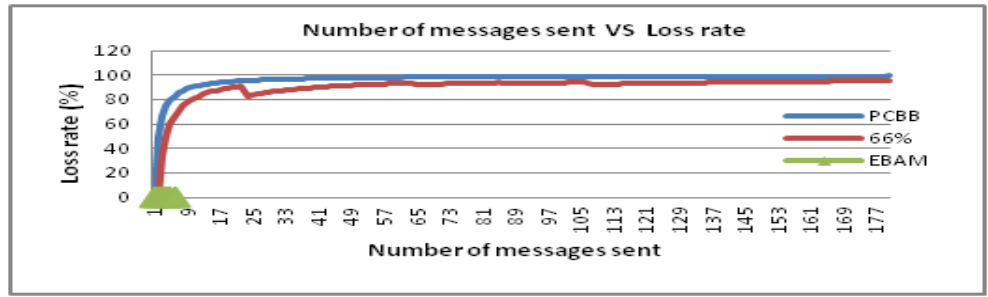

Fig. 8: Messages loss in the three protocols

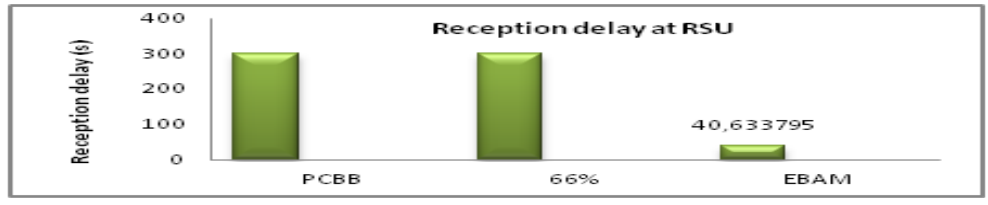

Fig. 9: Reception delay according to three protocols.

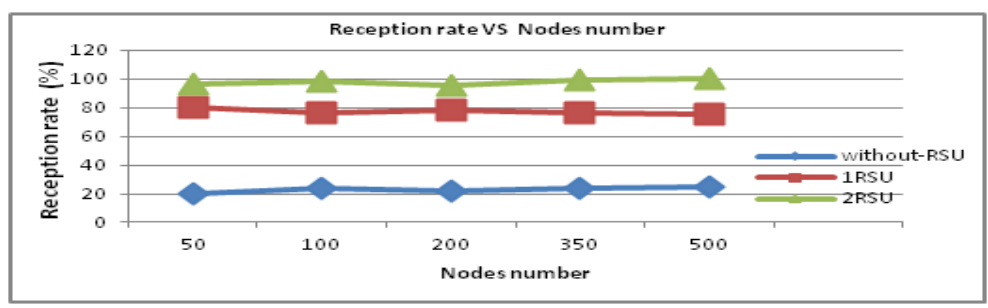

Fig. 10: Reception rate with 1 RSU, 2 RSU and without RSU.

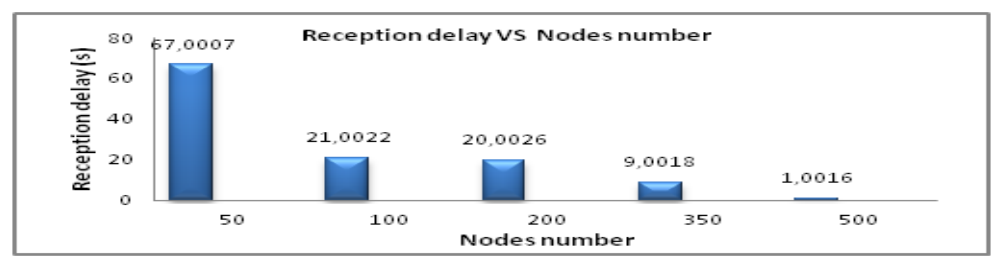

Fig. 11: Reception delay according to number of nodes.

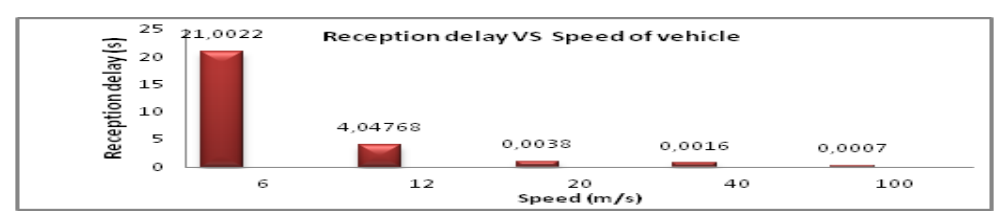

Fig. 12: Reception delay vs speed. 


\section{Conclusion}

In this paper, we studied the problem of efficient emergency message transmission in VANETs in order to propose a solution respecting dependable communications and specific time constraints of VANET environments. The EBAM proposed protocol showed an appreciable improvement of successfully delivered urgent messages in accordance with the real time constraint. This enhanced result obtained even at high network density and high node velocities is of great importance in order to well-timed reacting to crucial events (Like road accidents) by rescue services.

As a future work, it is desirable to increase the improvement of the proposal, by considering the vehicle directions using GPS.

\section{References}

1. Batish, S., Kakria, A.: Efficient broadcasting protocol for vehicular ad-hoc network. International Journal of Computer Applications 49(23) (2012)

2. Chen, Y.D., Shih, Y.P., Shih, K.P.: An emergency message dissemination protocol using n-way search with power control for vanets. In: Communications (ICC), 2015 IEEE International Conference on. pp. 3653-3658. IEEE (2015)

3. Kang, M., Virdaus, I.K., Shin, S., Lee, C.G.: Limited area broadcast for warning message delivery over vehicular ad-hoc networks. EURASIP Journal on Wireless Communications and Networking (1), 1-16 (2016)

4. Li, X., Hu, B.j., Chen, H., Ye, J.: A distance-aware safety-related message broadcasting algorithm for vehicular networks. International Journal of Distributed Sensor Networks 10(2), 139857 (2014)

5. Samara, G., Alhmiedat, T.: Intelligent emergency message broadcasting in vanet using pso. World of Computer Science and Information Technology Journal (WCSIT) ISSN: 2221-0741 4(7), 90-100 (2014)

6. Slavik, M., Mahgoub, I.: Spatial distribution and channel quality adaptive protocol for multihop wireless broadcast routing in vanet. IEEE Transactions on Mobile Computing 12(4), 722-734 (2013)

7. Torrent-Moreno, M., Jiang, D., Hartenstein, H.: Broadcast reception rates and effects of priority access in 802.11-based vehicular ad-hoc networks. In: Proceedings of the 1st ACM international workshop on Vehicular ad hoc networks. pp. 10-18. ACM, Philadelphia (october 2004)

8. Virdaus, I.K., Kang, M., Shin, S., Lee, C.G., Pyim, J.Y.: A counting-based broadcast model of emergency message dissemination in vanets. In: Ninth International Conference on Ubiquitous and Future Networks (ICUFN). pp. 927-930. IEEE (2017)

9. Yang, Y., Liu, Q., Gao, Z., Qiu, X., Rui, L., Li, X.: A data dissemination mechanism for motorway environment in vanets. EURASIP Journal on Wireless Communications and Networking 93(1) (2015) 